\title{
Effects of Teaching Classification on Classification, Verbal Conceptualization, and Analogical Reasoning in Children With Developmental Language Delays
}

\author{
David Tzuriel \\ Esther B. Isman \\ Tamar Klung \\ Bar-Ilan University, Ramat Gan, Israel \\ H. Carl Haywood \\ Vanderbilt University, Nashville, Tennessee
}

\begin{abstract}
Children, 4-6 years of age, in special education kindergartens were randomly assigned to a classification training $(n=45)$ and a comparison $(n=49)$ group. Children in the training group were taught the Classification unit of Bright Start, whereas those in the comparison group received a regular content-oriented curriculum. Both groups were given pre- and posttests of classification, semantic categories, and conceptual and perceptual analogies. Children who received the classification training improved more on all tests than did those in the comparison group. Significant positive correlations were found between verbal conceptualization and classification, conceptual analogies, and perceptual analogies. Teaching classification appears to have effects that generalize to other domains of language and higher order thinking that are significant in the cognitive development of young children with developmental language delays. The findings support the interplay between thinking and language and positive cognitive developmental effects of training in classification.
\end{abstract}

Keywords: classification; analogies; language delays; Bright Start

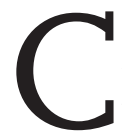

ontrol of linguistic knowledge commensurate with age is a condition for the ability to master spoken and written language (e.g., Korat \& Aram, 2011). Children who have difficulty acquiring linguistic knowledge typically also have difficulty learning to read and write. There are various sources of these difficulties, including genetic familial factors, socioeconomic factors, poor learning environments, and ineffective teaching methods (Ravid, Levie, \& Avivi-Ben Zvi, 2003). Leonard (2003) defined one manifestation of developmental language delays, specific language impairment (SLI), as a significant deficit in 
linguistic abilities with no evidence of primary difficulties such as hearing impairment, low intelligence, or emotional disorders. Hoffman and Gillam (2004) suggested that the origin of that disorder lies in deficits in perception, auditory processing, and structural linguistic input that make it difficult for children to generalize and link new information with preexisting information. Such children may have limited vocabulary, difficulty in retrieving words and defining them, reduced use of complex sentences, and poor narrative ability (Dockrell \& Messer, 2004). Researchers who have studied the ability of children to perform despite their impairments, whether congenital or environmental, have found that difficulties originating from congenital impairment are typically more consistent and less affected by the type of task and the complexity of the linguistic structure than are difficulties originating from environmental deprivation (Berman \& Ravid, 2009). Researchers agree that early detection of impairment, whether or not associated with a formal diagnostic category, is essential for building an intervention and mediation plan. The main objective of this study is to demonstrate that training for classification at early age applied with children with developmental language delays can facilitate not only classification ability (near transfer) but could be transferred to verbal conceptualization (medium transfer) and analogical reasoning (far transfer). In the following sections, we discuss briefly the concept of classification and its development of, the relation of classification and categorization to language acquisition, transfer of learning, the relation between classification and analogical reasoning, and the Bright Start program from which Classification unit was chosen for intervention.

\section{CLASSIFICATION AND ITS DEVELOPMENT}

Classification refers to cognitive action in which objects and events are grouped according to the logical relations governing their similarity. Classification allows one to impose order and control on a complex and often-chaotic world by identifying objects in our surrounding world, reducing a seemingly infinite number of individual objects, persons, places, or events to a more manageable number of logically related classes.

Preschool-age children first compare, abstracting similarities and differences, then categorize (grouping according to essential or pertinent similarities), trying thereby to find the "natural order" in the world (Gelman, 2003). Several researchers have found that categorization begins in infancy (Arterberry \& Bornstein, 2002; Bomba, 1984; Bomba \& Siqueland, 1983; Madole \& Oakes, 1999; Quinn, 1994; Younger, 1992). It starts with categorizing according to basic concepts (e.g., color, shape), which actions may be wholly perceptual ("these objects look alike," "all have four legs") without understanding of the logical relations among the categorized events.

\section{Differences Between Categorization and Classification}

Both Rosch, Mervis, Gray, Johnson, and Boyes-Braem (1976) and Jacob (2004) argue that the differences between classification and categorization are essential for construction of knowledge. The failure to distinguish between them appears to stem from the misconception that they are synonymous. This misconception may be reinforced by the fact that both are mechanisms for organizing information. Categorization was defined by Jacob (2004) as the process of dividing the world into groups of entities whose members are in some way similar to each other. Recognition of resemblance across entities and the subsequent aggregation of like entities into categories lead the individual to discover order in a complex environment. "Without the ability to group entities based on perceived similarities, the individual's 
experience of any one entity would be totally unique and could not be extended to subsequent encounters with similar entities in the environment" (p. 518). Unlike categorization, classification is a process involving systematic assignment of entities to one and only one class within a system of mutually exclusive and nonoverlapping classes. This process is lawful because it is carried out in accordance with an established set of principles that governs the structure of classes and class relationships. It is also systematic because it requires consistent application of these principles within the framework of a prescribed ordering of reality. Jacob identified six systemic properties for comparing systems of classification and categorization: (a) Process. Categorization is characterized by creative synthesis of entities based on context or perceived similarity, whereas classification is characterized by systematic arrangement of entities based on analysis of necessary and sufficient characteristics. (b) Boundaries. In categorization, boundaries are "fuzzy" because membership in a group is nonbinding, whereas in classification, boundaries are fixed because classes are mutually exclusive. (c) Membership. In categorization, membership is flexible, whereas in classification, it is rigorous; an entity either is or is not a member of a particular class. (d) Criteria for assignment. In categorization, the criteria for assignment is both context dependent and context independent, whereas in classification, it is predetermined by guidelines and principles. (e) Membership. In categorization, individual members of a category can be rank-ordered by typicality, whereas in classification, they are equally representative. (f) Structure. In classification, there is generally a hierarchical structure of well-defined, mutually exclusive, and nonoverlapping classes nested in a series of superordinate-subordinate systems, whereas categorization consists of variable clusters of entities that may or may not be organized in a hierarchical structure.

As children begin to conceptualize relations and to form verbal abstractions ("they are all wild animals"), the prior ability to categorize may develop into ability to classify based on conceptualized logical relations and develops into ability to classify events according to more complex and increasingly abstract criteria (Blaye, Bernard-Peyron, Paour, \& Bonthoux, 2006; Blaye, Chevalier, \& Paour, 2007; Bomba, 1984; Cèbe, Paour, \& Goigoux, 2002; Corrigan \& Denton, 1996; Mandler, 2000; Quinn \& Eimas, 1996). Thus, categorization precedes classification in the developmental sequence: comparison $\rightarrow$ categorization $\rightarrow$ conceptualization $\rightarrow$ classification.

\section{TRANSFER OF LEARNING}

Transfer of learning occurs when learning in one context or with one set of materials impacts on performance in another context or with other related materials. Transfer is a key concept in education and learning theory because most formal education aspires toward transfer. Consequently, the goals of education are not achieved unless transfer occurs and cannot be taken for granted. Evidence shows that very often the hoped-for transfer from learning experiences does not occur (Perkins \& Salomon, 1994).

Research findings indicate great difficulty in achieving distal transfer. In studies where transfer was achieved, it was limited, relative to levels of investment and expectation. To be achieved, transfer must become a central goal in teaching. Kaniel (2006) proposes that one should examine the products and processes of transfer from the learner's point of view. This perspective rests on a distinction between transfer potential, which relies on a network of schemas in memory storage, and the transfer process itself, which occurs in working memory. This analysis reduces the number of transfer characteristics and suggests description of transfer as an effective and justified use of principles, relationships, and strategies, in tasks that are distal from the learner's point of view. 


\section{Categorization, Classification, and Language Acquisition}

There is much debate over the relationship between thinking and language. According to Dromi (1986), the use of thinking categories allows children to learn new words rapidly. In this process, the acquisition of words is "horizontal," that is, acquisition of words that are attributed to different semantic groups. Typically, a single word appears in each category. For example, the word hat is acquired under the clothing category, and the word car is acquired under the vehicle category. When the child's vocabulary expands, there is a "vertical" acquisition, that is, acquisition of words that are related to the same category. For example, the words socks, trousers, and so forth will be added to the word hat under the clothing category. The transition from horizontal to vertical acquisition of words indicates the child's ability to recognize similarities and differences between the items and arrange them in distinct groups or categories (Dromi \& Fishelzon, 1986).

In the evolving literature, there are many approaches regarding the relationship between language and categorization among children. Diesendruck (2003) reviews three areas in which children are required to use categorization and generalization ability. When categorizing the animal domain, children's categorization processes are not significantly affected by the processes of conceptualization and culture in relation to their categorization of objects and people. His findings indicate that interplay exists between the area of children's specific cognitive abilities of categorization and their linguistic abilities.

While language acquisition takes place, children rely on existing knowledge. For example, Clark (2004) examined the actions of children in space, in the absence of relevant relation concepts. He found that children use existing conceptual categories for understanding new words and in construction of the relevant conceptual language. He claims that they construct their own language based on the categories that they have already acquired. The conceptual representation they have built in their first year of life for objects, relationships, property, and events provides a broad cognitive basis for their mapping of new words. In addition, cognitive research in nonlingual areas have already shown that children make generalizations and expand categories, provided that the new components possess significant and striking similarities to the components with which they are already familiar (Kotovsky \& Gentner, 1996).

Accordingly, it was found that children can make broad generalizations beyond their existing conceptual knowledge to create a new verbal category, provided that the basic similarities between the new word and the target word are significant and prominent enough. This capability is compatible with nonlinguistic research, and it is evident that language generalizations relate to the ability to make other cognitive generalizations (Goldberg, Casenhiser, $\&$ White, 2007). Beyond the linguistic implications regarding the categorization of language, this study provides evidence that knowledge acquired in various fields of cognitive psychology is able to clarify the language acquisition process in general and conceptualization processes in particular. Diesendruck's (2003) findings also indicate that there is interplay between the area of specific cognitive abilities of categorization and linguistic abilities.

\section{Classification and Analogical Reasoning}

The foregoing observations suggest that classification is fundamental to the development of relatively higher order cognitive operations and concepts. Green, Fugelsang, and Dunbar (2006) have already shown that activation of category concepts plays a fundamental role in 
analogical thinking. In this study, we sought to determine the extent to which improving classification skills might promote development of or improvement in analogical reasoning. The concept of analogy refers to recognizing relations between objects or events based on their similarities and transferring them to a different field (Kaniel, 2001). Each new concept formation means stretching an analogical link between different but familiar areas. Analogical reasoning is an important element in creating expectations and dealing with problem solving (Gentner, 1983). Analogical reasoning involves categorization, classification, and learning processes, which provide thinking tools that enable explanation for discovery and creative thinking (Goswami, 1992). Analogy is a very important element for enhancing broader thinking skills such as problem solving (Novick \& Basssok, 2005) and inductive thinking (Holland, Holyoak, Nisbett, \& Thagard, 1986) and provides a tool for thinking and scientific discoveries (Goswami, 1992).

\section{BRIGHT START: COGNITIVE CURRICULUM FOR YOUNG CHILDREN}

Bright Start (Brooks \& Haywood, 2003; Haywood, Brooks, \& Burns, 1992) is a cognitive education program primarily for preschool, kindergarten, and some primary-grade children. It has been implemented in several countries over the last 30 years and has been evaluated in several quantitative studies (e.g., Cèbe, 2000; Cèbe \& Paour, 2000; Paour, Cèbe, \& Haywood, 2000; Paour, Cèbe, Lagarrigue, \& Luiu, 1992; Tzuriel, Haywood, \& Mandel, 2005; Tzuriel, Kaniel, Kanner, \& Haywood, 1999; Tzuriel, Kaniel, Zeliger, Friedman, \& Haywood, 1998). The program is designed for children from 3 to 6 years of age and is focused on precognitive activities, cognition, and meta-cognition as prerequisites to academic learning. The program consists of eight curriculum units: Self-Regulation, Number Concepts, Comparison, Role Taking, Classification, Sequence and Pattern, Distinctive Features (letter-shape concepts), and Transformation. These are units that address the fundamental aspects of children's cognitive functioning and learning skills. Cognitive functions and processes are identified and emphasized in each lesson, and the teacher facilitates the children's application of these cognitive functions and processes in other contexts throughout the day, using generalization activities and bridging discussions within each lesson. For this study, we used only the Classification unit. This unit is aimed at developing the children's ability to classify objects, events, or persons according to the dimensions of color, size, shape, and number and to conceptualize the classifying criteria, leading to representational classification (i.e., classifying without referring to pictures or actual objects). The goals of this unit include children's learning to classify objects flexibly by different criteria and to provide rationales for their classification. The 16 lessons are administered in small groups of 3-6 children at a time. Each lesson takes about 20-30 min in which children learn through games, contests, "hands-on" (manipulative) experiences, and group discussion. Lessons are taught by a teacher trained in a mediational teaching style (Haywood, 1993, 2003) using mediated learning strategies (Feuerstein, Feuerstein, Falik, \& Rand, 2002). Cognitive functions that the Classification unit addresses include classification, class inclusion, verbal abstracting, comparison, discrimination of relevant information, grouping, labeling, being precise and accurate, seriation, use of spatial referents, and systematic exploration. For example, the main activity in Lesson 8 is to classify 10 pictures into groups of toys (5) and clothing (5) and assign the proper label for each. A generalizing activity is then applied when children are shown a picnic basket 
containing food and dishes. Each child is asked to remove one item from the basket, state a group to which it belongs, and put it in that group. A bridging discussion is followed to elicit and discuss other times when knowing the name of a group makes it easier to put similar things together. In addition, large group (whole class) lessons provide a framework for teaching specific academic content while simultaneously emphasizing the cognitive functions that are being taught directly in small group lessons. Thus, classification skills are further reinforced when they are being applied in varied domains. The content is not taught in isolated bits to be committed to memory for successful performance and repetition, but rather, the idea is to present content in a general context and to help children to generalize cognitive functions and cognitive strategies to everyday situations (Haywood et al., 1992). This unit of Bright Start was used to teach classification skills and concepts because it has been shown, both independently and as part of the larger curriculum, to yield impressive gains not only in classifying skills but also in general cognitive development, and its 16 lessons are focused sharply on classification.

In this study, we wished to assess the effects of teaching classification on developing classification skills, verbal conceptualization, and analogical reasoning in children with developmental language delays. These three skill domains represent near transfer, medium transfer, and far transfer, respectively. Another objective of this study was to determine whether classification skills are systematically related to verbal conceptualization and analogical reasoning abilities. Our classification test is made up of picture cards, which, according to Jacob (2004), have a very systematic arrangement of entities (process), the criteria are predetermined and based on very precise principles (criteria for assignment), and there is a hierarchical structure of fixed classes, such as in the case of animals, birds, and insects (structure), this make classes mutually exclusive and nonoverlapping (boundaries), and in the end, the child must decide if an entity is or is not a member of the particular class (membership).

\section{EXPECTATIONS}

1. Pre- to postintervention improvement in classification skills were expected to be greater for children who received training in classification than for children who did not. Change in classification skills represent near transfer, especially that this part of the test emphasizes categorization primarily.

2. Pre- to postintervention improvement in verbal conceptualization was expected to be greater for children in the classification training group than for those in the control group. Change in verbal conceptualization skills was taken to represent the medium transfer domain.

3. Pre- to postintervention improvement in analogical reasoning was expected to be higher among children in the classification training group compared with children in the control group. Change in analogical reasoning skills was taken to represent far transfer.

4. We expected to find significant positive correlations between verbal conceptualization and classification, conceptual analogies, and perceptual analogies. This expectation was based on research regarding classification skills and their influence on language acquisition processes in general and conceptualization processes in particular (Clark, 2004; Goldberg et al., 2007) and higher order thinking (Gentner \& Christie, 2010; Goldstone \& Hendrickson, 2010). 


\section{METHOD}

\section{Sample}

The sample comprised 94 Hebrew-speaking kindergarten children with developmental language delays, 4-6 years old, enrolled in kindergartens in Israel for children diagnosed with developmental language delays. The term developmental delay is applied when children do not achieve developmental milestones within the normal age range. All children in this sample had language or speech delays, which included the ability to communicate with other people effectively, express and receive information such as instructions on how to complete an assignment, and to form sentences. Some of the children had secondary developmental delays in motor, social, emotional, or intellectual development, although most were not diagnosed with secondary delays because of their young age. The children were randomly assigned to training $(n=45)$ and no-training $(n=49)$ groups. The training group, 30 boys and 15 girls, was taught in five different kindergartens by the kindergarten staff, all under the training and supervision of the same supervisor. Each staff member received $6 \mathrm{hr}$ of training. The staff used the Classification unit of Bright Start, as a supplemental part of their regular integrated thematic (content-oriented) curriculum. The control group, 35 boys and 14 girls, continued receiving the thematic curriculum. Both groups received the same amount of teaching time. To examine whether the experimental and control groups differed in pretreatment variables (classification, analogical reasoning, verbal conceptualization) and background variables (age, socioeconomic status, gender), we conducted a series of preliminary analyses. The analyses revealed no differences between groups in age, $t(92)=-0.32, p=.75$, socioeconomic status, $\chi^{2}(2)=3.95, n s$, or gender, $\chi^{2}(2)=0.71, n s$.

\section{Criterion Assessment}

Classification Test. The Classification Test (Tzuriel \& Stern, 2004) is composed of three stages, each stage scored separately, and includes cards of 13 main categories, represented by illustrations. This tool is a modified version of the Classification subtest of the Children's Inferential Thinking Modifiability test (Tzuriel, 1992). In each main category, there are four images that characterize the category. In this study, we used seven of these categories that belong to three classificatory domains: animals, plants, and inanimate objects. The items are typical for each category and are familiar to children. In the first stage, children are asked to sort the picture cards into groups, basically, a simple categorization task, with each correct grouping scored between 0 and 3 points, depending on the number of cards grouped correctly. In the second stage, children are asked to explain the groups' essentialness, and each answer is scored between 0 and 2 points, depending on the accuracy of the children's explanation. For example, a full answer with a score of 2 is "they all grow on trees." A partial answer is "we eat them" or "they have vitamins." In the final stage, children are asked to name the group, and each answer is scored between 0 and 2 points, depending on the accuracy of the child's answer. The correct name gets a score of 2; a similar or created word gets a score of 1 point. Scores are converted to percentages because each stage has a different scale. Tzuriel and Stern (2004) reported a Cronbach's alpha coefficient of .78 for the three scales. The Cronbach alpha based on the current sample was .76.

Katzenberger Assessment of Language in Preschool Age. This tool (Katzenberger, 2004) assesses spoken language in Hebrew. It is intended for children ages 4-6 years, comparing 
their performance to normal (age norms) linguistic development. It contains various aspects of linguistic ability, such as auditory processing, lexicon, morphological structures, phonological awareness, and syntax. The assessment is divided into seven sections, each section including a series of subtests. For this study, the semantic categories subtest was used because its content is moderately related to the tasks in the Bright Start Classification unit. This subtest examines the ability to categorize, which is part of the linguistic knowledge dimension. This ability is tested using semantic category, retrieval of words presented, and identifying a category that belongs to a group of words. This test yields scores on (a) speed of retrieval time (e.g., naming as many foods as possible in $1 \mathrm{~min}$ ), in which each correct answer scored 1 point; (b) categorical retrieval (e.g., naming two games or two musical instruments), in which each correct answer scored 1 point; and (c) similarity between objects (e.g., the similarity between a drum and a guitar). Each answer is scored between 0 and 2 points, depending on the accuracy of the children's explanation. No pictures are offered; the category membership is based on generalized knowledge. Participants' scores in each section are compared to age norms. The Cronbach's alpha coefficient based on the sample of this study was .69.

Children's Conceptual and Perceptual Analogical Modifiability Test-Closed Analogies Version. The Children's Conceptual and Perceptual Analogical Modifiability Test (CCPAM)Closed Analogies Version (Tzuriel, 2003, 2007) is a dynamic assessment instrument for examining children's ability to solve conceptual and perceptual analogies. It is composed of two sets of analogical problems, conceptual (16 items) and perceptual (20 items). Each problem is formatted in a $2 \times 2$ matrix (A: B :: C: D) and presented in a pictorial-colored modality at the top of the page. At the bottom of the page, there are four alternative answers, only one of which is the correct answer. The children are required to think about the relationship between the first pair of pictures in the problem, apply that relation to the second pair, and choose the correct answer from the four alternatives. An example for a conceptual analogy is bird: nest :: dog : kennel. An example of a perceptual analogy is cat in purple box: cat outside of purple box :: cat in green box : cat outside of green box. Each problem in the preteaching phase parallels a problem in the postteaching phase, in terms of the relation expressed in the analogy; both analogies exemplifying the same relationship. In each subtest, two analogies are used for instruction and rule explanation before administration of the test. Each correctly solved analogy gets a score of 1 . The maximal scores for the conceptual and perceptual subtests are 16 and 20, respectively. The Cronbach's alpha reliability coefficients for the preteaching conceptual analogies subtest ranged between .64 and .74 and for the postteaching, between .70 and .85 . The reliability coefficients of the preteaching perceptual analogies subtest ranged between .83 and .87 and for the postteaching, between .90 and .91. Internal consistency in this study as determined by Cronbach's alpha was .77. The validity of the CCPAM test has been demonstrated in several studies (e.g., Lifshitz, Tzuriel, \& Weiss, 2005; Lifshitz, Weiss, Tzuriel, \& Tzemach, 2010; Tzuriel, 2007; Tzuriel \& Flor-Maduel, 2010; Tzuriel \& George, 2009; Tzuriel \& Shamir, 2010; Vakil, Lifshitz, Tzuriel, Weiss, \& Arzuoan, 2011).

Demographic-Personal Questionnaire. The questionnaire, filled out by the teacher, calls for personal information about age, gender, parents' occupation, and number of siblings.

\section{Procedure}

All participants were given the CCPAM test, Katzenberger's Semantic Categories Subtest, and the Classification Test, in that order, before and after the intervention program. All tests were administered individually in a quiet room in the kindergarten. The Classification unit 
of Bright Start was applied in 16 sessions during 2 months of the school year. Children in the training group received the Bright Start training twice a week for 20 min each time in small groups of five or six children. The program was a part of each participant's individualized education plan. The children from the control group, instead of the classification training, continued with their regular content-oriented kindergarten curriculum. This curriculum, designed by the Ministry of Education for children with developmental language delays at kindergarten, is focused on language skills. Postintervention testing was done for both groups 2 months after the intervention ended.

\section{RESULTS}

Preliminary analyses did not show any group differences at the preintervention phase on Classification, $t(92)=.67$, ns; Analogical Reasoning, $t(92)=-1.59$, ns; or Verbal Conceptualization, $t(92)=-0.43$, $n$ s.

\section{Classification Skills, Verbal Conceptualization, and Analogical Reasoning in the Experimental and Control Groups}

To examine for differential change in classification skills (categorization sorting, essentialness, and naming), we conducted a repeated-measures multivariate analysis of variance (MANOVA) of Treatment $\times$ Time $\times$ Type of Skill $(2 \times 2 \times 3)$ where Treatment was an independent between-groups variable and the last two variables were within-subject independent variables. The dependent variables were the scores in percentages. The means and standard deviations are presented in Table 1.

The analysis revealed a significant main effect of Type of Skill, $F(2,91)=53.11, p<.001$, $\eta_{p}{ }^{2}=.37$. Sidak post hoc analyses indicated that participants achieved the highest scores on categorization sorting $(M=62.19, S E=2.42)$, followed by naming $(M=48.01, S E=2.23)$ and essentialness $(M=24.99, S E=1.99)$. The skill types were significantly different from each other in both treatment groups $(p<.001)$. The analysis also revealed a main effect of group, $F(1,91)=6.77, p=.011, \eta_{p}^{2}=.07$, indicating that participants in the training group achieved higher classification scores $(M=49.51, S E=2.47)$ than did those in the control group $(M=$ $40.61, S E=2.37)$. There was also a main effect of Time, $F(1,91)=26.10, p<.001, \eta_{p}{ }^{2}=.22$, indicating that postintervention scores on classification $(M=49.38, S E=1.96)$ were higher than preintervention scores $(M=40.75, S E=1.86)$. The main effects of Group and Time were moderated by an interaction of Group by Time, $F(1,91)=14.40, p<.001, \eta_{p}{ }^{2}=.14$.

TABLE 1. Means and Standard Deviations of Pre- and Postintervention Classification Skills in the Experimental and Control Groups $(n=96)$

\begin{tabular}{|c|c|c|c|c|c|c|c|c|}
\hline & \multicolumn{4}{|c|}{ Experimental } & \multicolumn{4}{|c|}{ Control } \\
\hline & \multicolumn{2}{|c|}{ Pre } & \multicolumn{2}{|c|}{ Post } & \multicolumn{2}{|c|}{ Pre } & \multicolumn{2}{|c|}{ Post } \\
\hline & $M$ & $S D$ & $M$ & $S D$ & $M$ & $S D$ & $M$ & $S D$ \\
\hline Categorization sorting & 61.05 & 26.32 & 76.83 & 21.26 & 52.48 & 30.27 & 58.41 & 25.93 \\
\hline Essentialness & 16.19 & 20.85 & 31.42 & 23.66 & 28.13 & 20.28 & 24.20 & 24.95 \\
\hline Naming & 48.73 & 21.17 & 62.86 & 21.99 & 37.91 & 26.89 & 42.57 & 25.24 \\
\hline
\end{tabular}




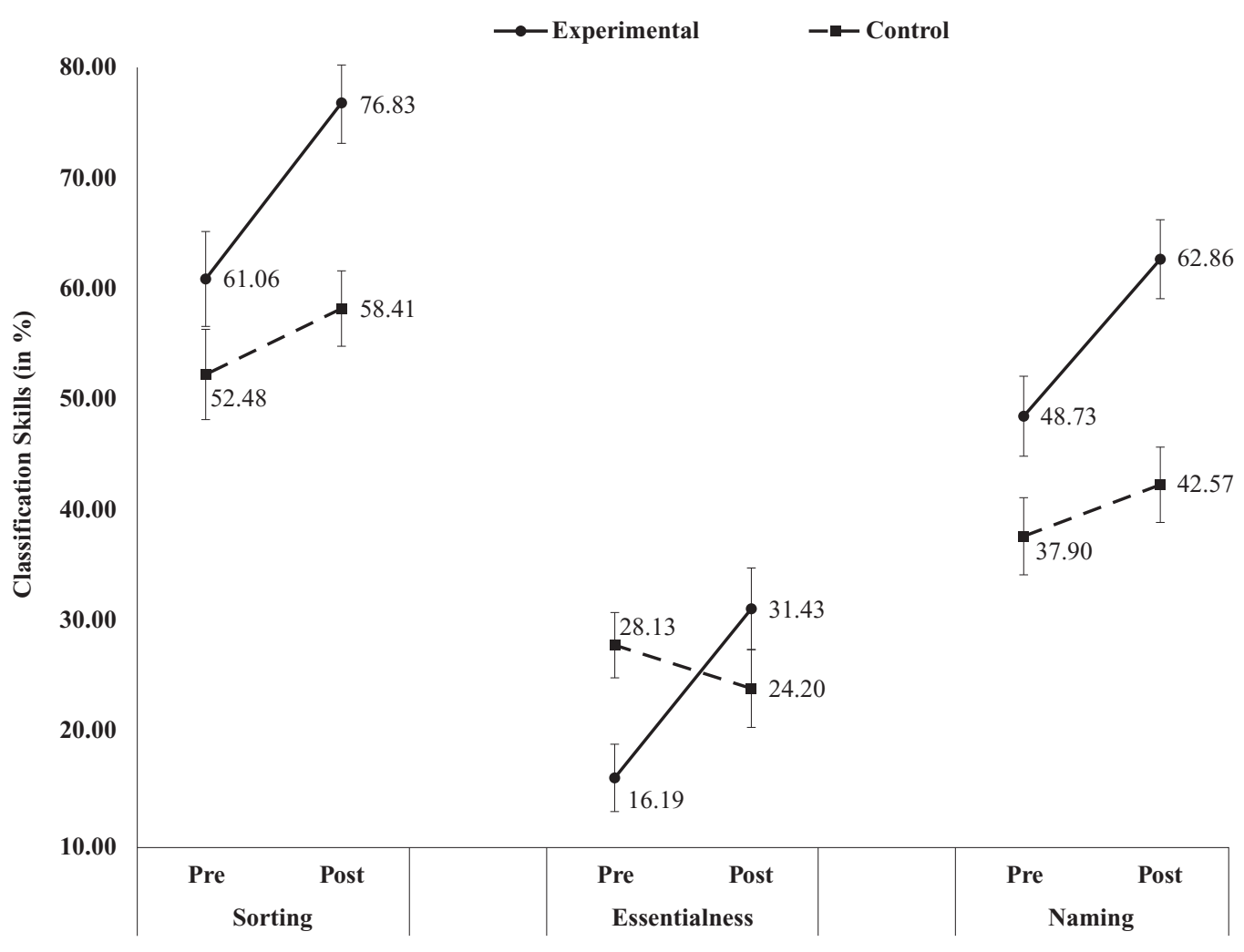

FIGURE 1. Pre- and postintervention scores in classification skills.

Simple effects tests with Bonferroni adjustment indicated that classification scores increased significantly in the training group $(p<.001)$ but not in the control group. Other effects were not significant. Figure 1 portrays the significant interaction.

\section{Pre- to Postintervention Change in Verbal Conceptualization}

To examine the pre- to postintervention change in verbal conceptualization (i.e., verbal retrieval time, verbal categorical retrieval time, verbal object similarity) and whether the treatment affected it differentially, we conducted repeated-measures MANOVA of Treatment by Time by Type of Skill, the last two variables being within-subject independent variables. The dependent variable was the score on these tests expressed in percentages of total possible score. The means and standard deviations are presented in Table 2.

This analysis revealed a significant main effect of Type of Skill, $F(2,91)=73.10, p<.001$, $\eta_{p}{ }^{2}=.44$. Sidak post hoc analyses indicated that participants achieved the highest scores on verbal retrieval time $(M=78.72, S E=2.02)$, followed by categorical retrieval time $(M=73.04$, $S E=2.24)$ and verbal object similarity $(M=60.50, S E=2.42$; all $p s<.01)$. The analysis also revealed a significant main effect of Time, $F(1,91)=36.85, p<.001, \eta_{p}{ }^{2}=.29$, indicating that postintervention scores on verbal conceptualization $(M=74.66, S E=1.97)$ were higher than preintervention scores $(M=66.85, S E=2.12)$. The main effect of Time was moderated by a Treatment by Time interaction, $F(1,91)=21.16, p<.001, \eta_{p}{ }^{2}=.19$. Simple effects tests with 
TABLE 2. Means and Standard Deviations of Verbal Conceptualization Scores by Treatment and Time $(n=96)$

\begin{tabular}{|c|c|c|c|c|c|c|c|c|}
\hline & \multicolumn{4}{|c|}{ Experimental } & \multicolumn{4}{|c|}{ Control } \\
\hline & \multicolumn{2}{|c|}{ Pre } & \multicolumn{2}{|c|}{ Post } & \multicolumn{2}{|c|}{ Pre } & \multicolumn{2}{|c|}{ Post } \\
\hline & $M$ & $S D$ & $M$ & $S D$ & $M$ & $S D$ & $M$ & $S D$ \\
\hline $\begin{array}{l}\text { Verbal retrieval } \\
\text { time }\end{array}$ & 75.87 & 24.47 & 86.51 & 19.87 & 76.82 & 22.71 & 75.65 & 20.09 \\
\hline $\begin{array}{l}\text { Categorical } \\
\text { retrieval time }\end{array}$ & 65.93 & 25.30 & 82.04 & 18.97 & 65.93 & 24.42 & 73.13 & 24.42 \\
\hline $\begin{array}{l}\text { Verbal object } \\
\text { similarity }\end{array}$ & 56.02 & 27.40 & 70.46 & 23.90 & 55.36 & 26.20 & 60.16 & 26.89 \\
\hline
\end{tabular}

Bonferroni adjustment indicated that verbal conceptualization skills significantly increased in the experimental group $(p<.001)$ but not in the control group. Figure 2 portrays these relationships.

\section{Pre- to Postintervention Improvement in Analogical Reasoning}

To examine the pre- to postintervention change in analogical reasoning and whether the treatment affected it differentially, we conducted repeated-measures MANOVA of Treatment by Time by Type of Analogy, the last two variables being within-subject independent variables.

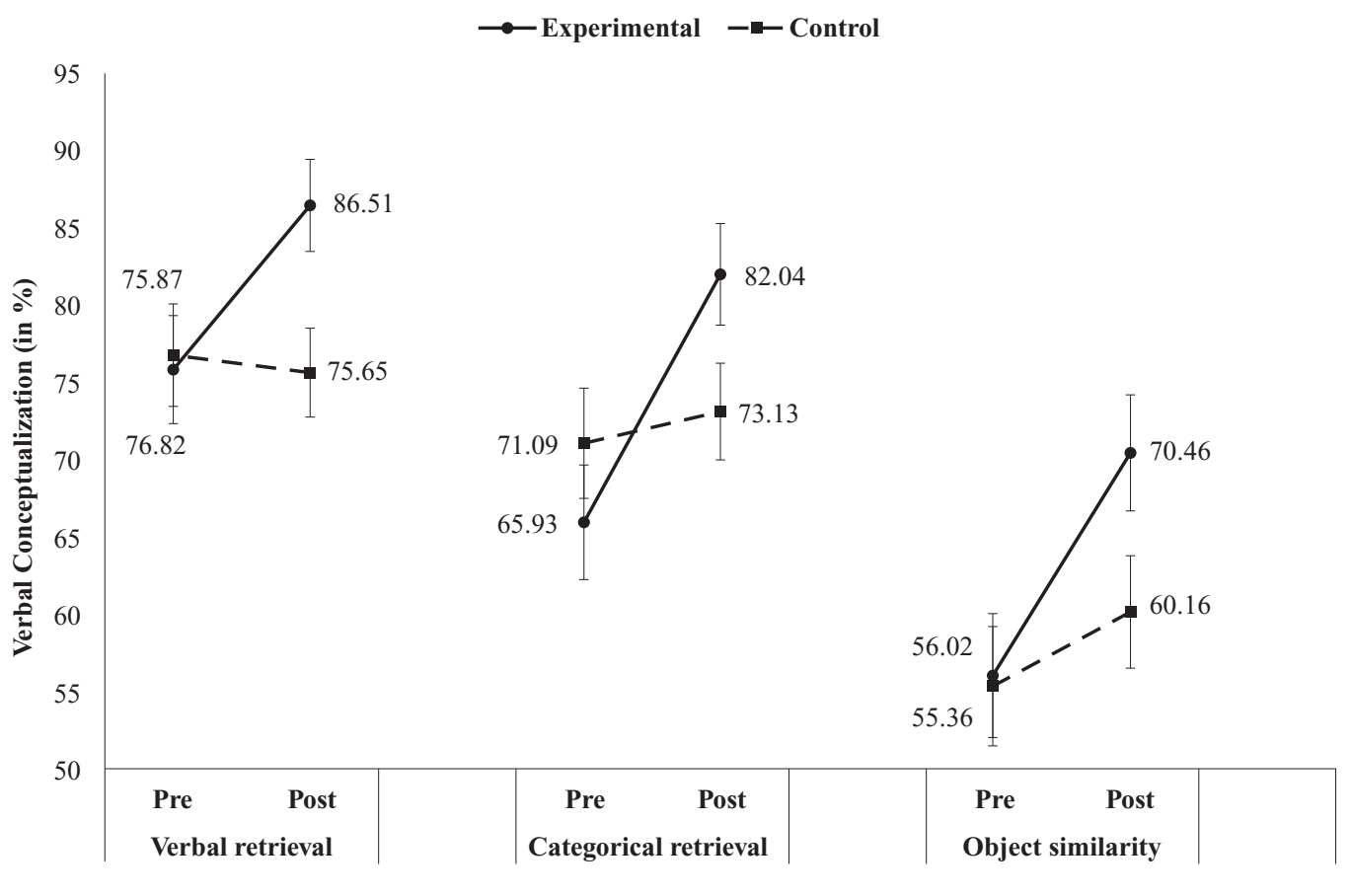

FIGURE 2. Pre- and postintervention scores in verbal conceptualization skills. 
TABLE 3. Means and Standard Deviations of Analogical Reasoning by Treatment and Time $(n=96)$

\begin{tabular}{|c|c|c|c|c|c|c|c|c|}
\hline & \multicolumn{4}{|c|}{ Experimental } & \multicolumn{4}{|c|}{ Control } \\
\hline & \multicolumn{2}{|c|}{ Pre } & \multicolumn{2}{|c|}{ Post } & \multicolumn{2}{|c|}{ Pre } & \multicolumn{2}{|c|}{ Post } \\
\hline & $M$ & $S D$ & $M$ & $S D$ & $M$ & $S D$ & $M$ & $S D$ \\
\hline Conceptual & 38.00 & 13.37 & 44.00 & 21.28 & 43.06 & 13.91 & 49.29 & 19.74 \\
\hline Perceptual & 35.14 & 22.31 & 54.03 & 25.88 & 40.18 & 21.42 & 48.47 & 20.67 \\
\hline
\end{tabular}

The dependent variable was the analogical reasoning score expressed in percentage of total possible score. The means and standard deviations are presented in Table 3.

The analysis revealed a significant main effect of Time, $F(1,91)=40.48, p<.001, \eta_{p}{ }^{2}$ $=.31$, indicating that the preintervention analogical reasoning scores $(M=39.10, S E=$ 1.58) were lower than postintervention scores $(M=48.95, S E=2.03)$. The main effect was modified by the interaction of Type of Analogy and Time, $F(1,91)=9.72, p=.002, \eta_{p}{ }^{2}=.10$. Simple effects test with Bonferroni adjustment indicated that perceptual analogies scores increased significantly from pre- to postintervention $(p<.02)$, whereas no significant changes were found for conceptual analogies. The two-way interaction was further modified by the triadic interaction of Treatment by Type of Analogy by Time, $F(1,91)=5.09, p=.026, \eta_{p}{ }^{2}=.05$. Figure 3 portrays these effects.

Simple effects tests with Bonferroni adjustment revealed a significant effect of Group $\times$ Time for perceptual analogies, $F(1,91)=6.48, p=.01, \eta_{p}{ }^{2}=.07$, but not for conceptual analogies, $F(1,91)=0.04, p=.95, \eta_{p}{ }^{2}=.00$. These findings indicate that although perceptual analogies scores increased for children in both groups (experimental and control), the magnitude of increase was significantly greater in the experimental group $(p<.001)$ than in the control group $(p=.005)$. Other effects were not significant.

\section{The Relation Between Verbal Conceptualization, Classification Skills, and Analogical Reasoning}

To examine whether classification, conceptual analogies, and perceptual analogies abilities were systematically related to verbal conceptualization, we combined the pre- and postintervention scores for all children and conducted a series of Pearson product-moment correlations for the whole sample. The analyses revealed significant positive correlations between verbal conceptualization and classification, $r(92)=.58, p<.001$; conceptual analogies, $r(92)=.37, p<.001$; and perceptual analogies, $r(92)=.31, p=.003$.

\section{DISCUSSION}

The purpose of this study was to examine the effects of teaching classification as represented in the Bright Start program (Haywood et al., 1992) on classification skills, verbal conceptualization, and analogical reasoning in children with developmental language delays. These three domains were chosen to represent near transfer, medium transfer, and far transfer effects, respectively. We also wished to determine whether verbal conceptualization is associated with classification skills and analogical reasoning abilities. Previous research has 


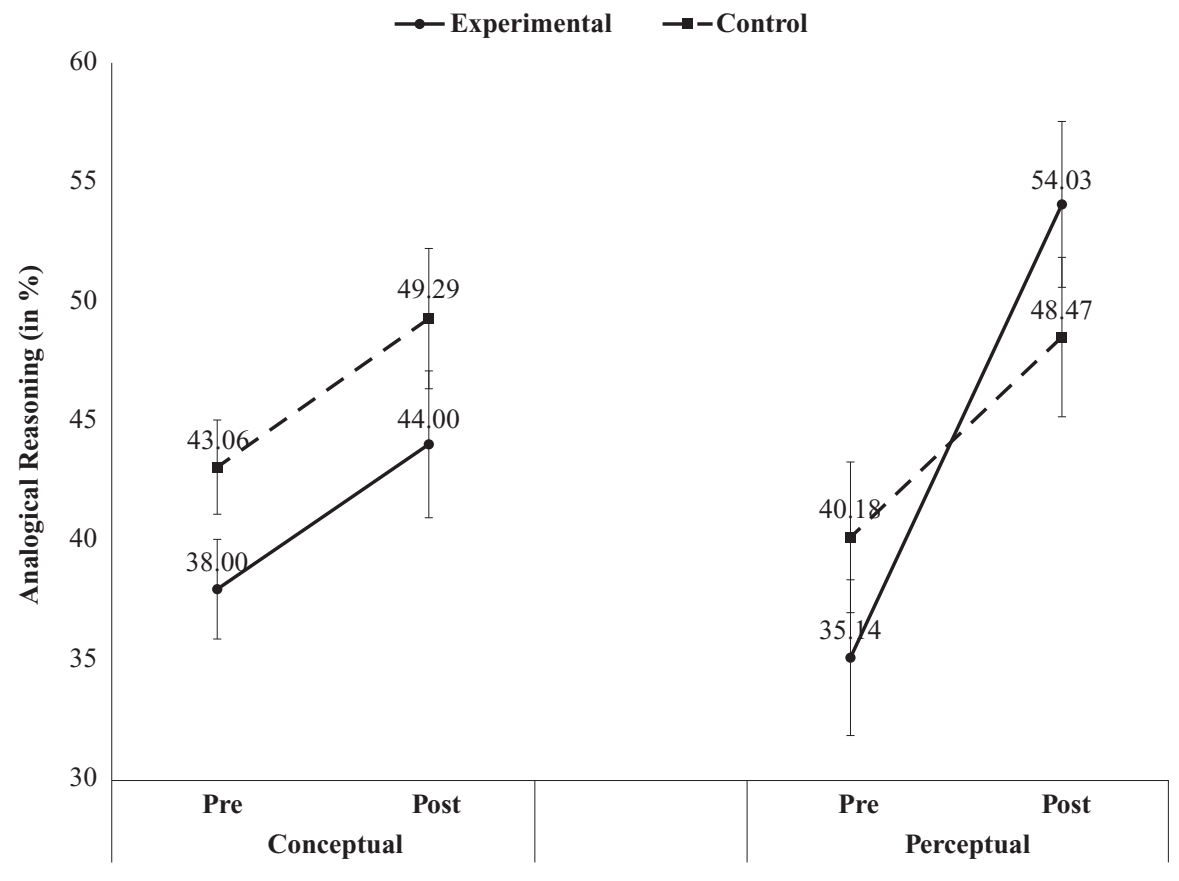

FIGURE 3. Pre- and postintervention scores in analogical reasoning.

revealed a significant association between classification skills and language acquisition processes (Goldberg et al., 2007). Gentner and Christie (2010) identified categorization and classification abilities as basic cognitive thinking skills that allow creative thinking and analogical reasoning.

Based on previous research, we expected that children trained in classification would improve in these three domains more than would children not so trained. In this study, children from both groups improved scores on most of the cognitive tests from pre- to postintervention, but the group trained in classification showed greater improvement than did the nontrained group. (The improvement in the nontrained group could reflect a combined effect of maturation and practice on the tests.) This finding accords with previous research (e.g., Clark, 2004; Diesendruck, 2003; Dromi, 1986, 2002; Dromi \& Fishelzon, 1986) and theory (e.g., Skinner, 1957; Vygotsky, 1934/1978) regarding the association between development of thinking and language skills. Furthermore, the results coincide with evidence of neuroplasticity of the brain because of category learning (e.g., Goldstone \& Hendrickson, 2010) and enhances our understanding of the role of classification in the development of both perceptual and conceptual abilities.

\section{Differences Among Classification Abilities}

The most difficult component of the Classification Test was essentialness, followed by naming and categorization sorting (see Table 1 and Figure 1). The most demanding and abstract mental activity required in classification is finding the essential feature that is common for the four elements and verbalizing it correctly. Children might have an intuitive understanding of the category and would sort and categorize elements of a group correctly but might have 
difficulty understanding the abstract unique essence of the grouping, hence the lower scores in this component in both the trained and nontrained groups. The relatively higher scores on the naming component (third stage of the test) could be related to the fact that it was given after the activity of finding the essential characteristic of the group and the fact that it is much easier to provide a name to a group than to explain the essence of the commonality. It is also interesting to note that whereas the nontrained group showed an increase in performance in categorization sorting and in naming from pre- to postintervention, it showed a decrease in the essentialness component, whereas the trained group showed an increase. Thus, the Bright Start training was most effective in the component that is, empirically, the most difficult. The difference between the two groups can be attributed to the abstract classification activities given in the training.

Goldstone and Hendrickson (2010) reported that both object-to-label associations (considered as low-level cognitive functions) and decision making (considered as a high-level cognitive function) are influenced by category learning. Similarly, Goldberg et al. (2007) have shown that language generalizations relate to the ability to make other cognitive generalizations as well as making broad generalizations beyond their existing conceptual knowledge. Paour et al. (1992) applied two Bright Start curriculum units, Self-Regulation and Classification, with preschool and kindergarten children, most of them from immigrant families in the south of France, and assessed the effects on tests of classification and several other cognitive and academic performance variables. They found marked positive effects on the children's performance on classification tasks, indicating that classification is responsive to training in young children, as well as a dramatic positive effect on the children's reading of novel words.

Children who received classification training showed higher pre- to posttraining improvement than did the nontrained group on perceptual analogies but not on conceptual analogies. A possible explanation for the differential effects of classification training on the two types of analogy is to be found in the characteristics of the analogies. Conceptual analogies, by definition, are more abstract than are perceptual analogies and require understanding of higher order concepts, principles, and relations (Lifshitz et al., 2005; Lifshitz et al., 2010; Tzuriel, 2007; Vakil et al., 2011). The short-term intervention in this study with kindergarten children with developmental language delays was sufficient to improve performance of concrete perceptual analogies but was not transferred to the abstract conceptual analogies. Nevertheless, these children transcended the barriers of young age and cognitive delays and improved their analogical thinking. Furthermore, a normative sample of typically developing children $(n=64)$ of the same age $(M=5: 6)$ in Tzuriel's (2007) study achieved a score of $35.95 \%$ in perceptual posttesting analogies as compared with $54.03 \%$ of the current sample of children with developmental language delays participating in the intervention. The higher analogical thinking of these children can be attributed to the teaching and learning of classification. Another possible explanation for the difference in effects on perceptual and conceptual analogies is the observation that classification, as contrasted with categorization, entails practice in conceptualizing and specifying the logical relations that govern construction and expansion of classes. It is likely that the Classification unit of Bright Start emphasizes categorization more heavily than classification, leaving responsibility for conceptualization and explicitation (see Bruderlein, 2004, for a discussion of the role of explicitation) of such relations largely in the hands of the teachers. Thus, effects of the classification training might quite reasonably be principally at the perceptual level. 
We wished further to examine the associations between language and higher level thinking. We found significant positive correlations between verbal conceptualization and classification skills and analogical reasoning. These findings are supported by contemporary research indicating the existence of interplay between the cognitive abilities of categorization, classification, and linguistic abilities (Diesendruck, 2003) as well as between categorization and analogical reasoning (Gentner \& Christie, 2010). A possible implication of this finding is that intervention for classification may transfer to other cognitive domains of language and higher order thinking skills such as analogical reasoning.

Although the objectives of the study were achieved and have led to the conclusion that the Bright Start Classification unit yields gains in the cognitive and linguistic functioning of young children with developmental language delays and led to near, medium, and far transfer, there are some limitations to this study that must be addressed. One limitation is the lack of follow-up of the effects of the intervention program. Another limitation is the relatively brief duration and mild intensity of the program-16 sessions of 20-30 min, thus a maximum of $8 \mathrm{hr}$ of training. It might well be that the effects of the program will fade out with time. We suggest therefore in future research to implement the program with different levels of length and intensity and carry out follow-up assessment at different time intervals. Further studies may examine the continuous development of the preschoolers as they enter first grade. Follow-up is needed to determine whether lessons taught in the Classification unit of Bright Start have lasting effects on children's cognitive and language development, as previous researchers have found (e.g., Cèbe \& Paour, 2000; Paour et al., 2000).

In addition to these considerations, it is important to examine whether the findings can be generalized to typical preschool children as well as other children with special educational needs, such as those formally diagnosed with SLI, autism spectrum disorders, intellectual disabilities, and sensory, especially hearing, impairment.

\section{REFERENCES}

Arterberry, M. E., \& Bornstein, M. H. (2002). Variability and its sources in infant categorization. Infant Behavior \& Development, 25, 515-528.

Berman, R. A., \& Ravid, D. (2009). Becoming a literate language user: Oral and written text construction across adolescence. In D. R. Olson \& N. Torrance (Eds.), The Cambridge handbook of literacy (pp. 92-111). New York, NY: Cambridge University Press.

Blaye, A., Bernard-Peyron, V., Paour, J.-L., \& Bonthoux, F. (2006). Categorical flexibility in children: Distinguishing response flexibility from conceptual flexibility: The protracted development of taxonomic representations. European Journal of Developmental Psychology, 3, 163-188.

Blaye, A., Chevalier, N., \& Paour, J.-L. (2007). The development of intentional control of categorization behavior: A study of children's relational flexibility. Cognition, Brain, \& Behavior, 11, 791-808.

Bomba, P. C. (1984). The development of orientation categories between 2 and 4 months of age. Journal of Experimental Child Psychology, 37, 609-636.

Bomba, P. C., \& Siqueland, E. R. (1983). The nature and structure of infant form categories. Journal of Experimental Child Psychology, 35, 294-328.

Brooks, P. H., \& Haywood, H. C. (2003). A preschool mediational context: The Bright Start curriculum. In A. S. S. Hoon, L. P. K. Hoon, \& O-S. Tan (Eds.), Mediated learning experience with children: Applications across contexts (pp. 98-132). Singapore, Singapore: McGraw-Hill.

Bruderlein, P. (2004). Explicitation and intellectual efficiency. Journal of Cognitive Education and Psychology, 4(2), 232-244. 
Cèbe, S. (2000). Déveloper la conceptualisation et la prise de conscience métacognitive à lécole maternelle: Effets sur l'efficience scolaire ultérieure du CP au CÉ2: Une contribution à la prévention de l'échec scolaire des élèves de milieux populaires [Developing conceptualization and metacognitive awareness in preschool: Effects on subsequent school achievement from first to third grades. A contribution to the prevention of school failure of pupils from low socio-economic level families] (Doctoral dissertation). Université de Provence, Aix-en-Provence, France.

Cèbe, S., \& Paour, J.-L. (2000). Effects of cognitive education in kindergarten on learning to read in the primary grades. Journal of Cognitive Education and Psychology, 1, 177-200.

Cèbe, S., Paour, J.-L., \& Goigoux, R. (2002). Catégo: Un imagier pour apprendre à catégoriser [A picture book for learning to categorize]. Paris, France: Hatier.

Clark, E. V. (2004). How language acquisition builds on cognitive development. Trends in Cognitive Sciences, 8, 472-478.

Corrigan, R., \& Denton, P. (1996). Casual understanding as a developmental primitive. Developmental Review, 16, 162-202.

Diesendruck, G. (2003). Categories for names or names for categories? The interplay between domainspecific conceptual structure and language. Language and Cognitive Processes, 18, 759-787.

Dockrell, J. E., \& Messer, D. A. (2004). Lexical acquisition in the early school years. In R. A. Berman. (Ed.), Language development across childhood and adolescence (pp. 35-52). Philadelphia, PA: J. Benjamins.

Dromi, E. (1986). The one-word period as a stage in language development: Quantitative and qualitative accounts. In I. Levin (Ed.), Stage and structure: Reopening the debate (pp. 220-245). Norwood, NJ: Ablex.

Dromi, E. (2002). Stages in the acquisition of Hebrew as a mother tongue. In P. S. Klein \& D. Givon (Eds.), Language, learning and literacy in the preschool years (pp. 9-41). Tel-Aviv, Israel: Ramot.

Dromi, E., \& Fishelzon, G. I. (1986). Similarity, specificity and contrast: A study of early semantic categories. Papers and Reports on Child Language Development, 25, 25-32.

Feuerstein, R., Feuerstein, R. S., Falik, L. H., \& Rand, Y. (2002). The dynamic assessment of cognitive modifiability: The Learning Propensity Assessment Device: Theory, instruments, and techniques. Jerusalem, Israel: International Centre for the Enhancement of Learning Potential.

Gelman, S. A. (2003). The essential child: Origins of essentialism in everyday thought. New York, NY: Oxford University Press.

Gentner, D. (1983). Structure mapping: A theoretical framework for analogy. Cognitive Science, 7, $155-170$.

Gentner, D., \& Christie, S. (2010). Mutual bootstrapping between language and analogical processing. Language and Cognition, 2, 261-283.

Goldberg, A. E., Casenhiser, D., \& White, T. R. (2007). Constructions as categories of language. New Ideas in Psychology, 25, 70-86.

Goldstone, R. L., \& Hendrickson, A. T. (2010). Categorical perception. WIREs Cognitive Science, 1, 69-78.

Goswami, U. (1992). Analogical reasoning in children. Mahwah, NJ: Lawrence Erlbaum Associates.

Green, A. E., Fugelsang, J. A., Dunbar, K. N. (2006). Automatic activation of categorical and abstract analogical relations in analogical reasoning. Memory \& Cognition, 34, 1414-1421. http://dx.doi.org/ proxy1.athensams.net/10.3758/BF03195906

Haywood, H. C. (1993). A mediational teaching style. International Journal of Cognitive Education and Mediated Learning, 3(1), 27-38.

Haywood, H. C. (2003). Mediation within a neo-Piagetian framework. Journal of Cognitive Education and Psychology, 3(1), 71-81.

Haywood, H. C., Brooks, P. H., \& Burns, M. S. (1992). Bright Start: Cognitive curriculum for young children. Watertown, MA: Charlesbridge.

Hoffman, L. M., \& Gillam, R. B. (2004). Verbal and spatial information processing constraints in children with specific language impairment. Journal of Speech, Language, and Hearing Research, 47, 114-125. http://dx.doi.org/10.1044/1092-4388(2004/011) 
Holland, J. H., Holyoak, K. J., Nisbett, R. E., \& Thagard, P. R. (1986). Induction: Processes of inference, learning and discovery. Boston, MA: MIT Press.

Jacob, E. K. (2004). Classification and categorization: A difference that makes a difference. Library Trends, 52, 515-540.

Kaniel, S. (2001). The psychology of control of cognition. Ramat Gan, Israel: Bar-Ilan University.

Kaniel, S. (2006). Teaching for thinking: Cognitive education towards controlling the mind. Tel Aviv, Israel: Center for Educational Technology.

Katzenberger, I. (2004). Katzenberger Hebrew language assessment for pre-school children. Jerusalem, Israel: Hadassah Academic College.

Korat, O., \& Aram, D. (2011). Literacy and language: Relationship, bilingualism and difficulties. Jerusalem, Israel: Magnes.

Kotovsky, L., \& Gentner, D. (1996). Comparison and categorization in the development of relational similarity. Child Development, 67, 2797-2822.

Leonard, L. B. (2003). Specific language impairment: Characterizing the deficit. In Y. Levy \& J. Schaeffer (Eds.), Language competence across populations: Toward a definition of specific language impairment (pp. 209-231). Mahwah, NJ: Lawrence Erlbaum Associates.

Lifshitz, H., Tzuriel, D., \& Weiss, I. (2005). Effects of training in conceptual versus perceptual analogies among adolescents and adults with intellectual disability. Journal of Cognitive Education and Psychology, 5, 144-167.

Lifshitz, H., Weiss, I., Tzuriel, D., \& Tzemach, M. (2010). New model of mapping difficulties in solving analogical problems among adolescents and adults with intellectual disability. Research in Developmental Disabilities, 32, 326-344.

Madole, K. L., \& Oakes, L. M. (1999). Making sense of infant categorization: Stable processes and changing representations. Developmental Review, 19, 263-296.

Mandler, J. M. (2000). Perceptual and conceptual processes in infancy. Journal of Cognition and Development, 1, 3-36.

Novick, L. R., \& Bassok, M. (2005). Problem solving. In K. J. Holyoak \& R. G. Morrison (Eds.), The Cambridge handbook of thinking and reasoning (pp. 321-350). Cambridge, United Kingdom: Cambridge University Press.

Paour, J.-L., Cèbe, S., \& Haywood, H. C. (2000). Learning to learn in preschool education. Journal of Cognitive Education and Psychology, 1, 3-25.

Paour, J.-L., Cèbe, S., Lagarrigue, P., \& Luiu, D. (1992). A partial evaluation of Bright Start with pupils at risk of school failure. The Thinking Teacher, 8, 1-7.

Perkins, D. N., \& Salomon, G. (1994). Transfer of learning. In T. Husen \& T. N. Postlethwaite (Eds.), The international encyclopedia of education (2nd ed., pp. 6452-6457). Oxford, United Kingdom: Pergamon.

Quinn, P. C. (1994). The categorization of above and below spatial relations by young infants. Child Development, 65, 58-69.

Quinn, P. C., \& Eimas, P. D. (1996). Perceptual organization and categorization in young infants. In C. Rovee-Collier \& L. P. Lipsitt (Eds.), Advances in infancy research (Vol. 10, pp. 1-36). Norwood, NJ: Ablex.

Ravid, D., Levie, R., \& Avivi-Ben Zvi, G. (2003). Morphological disorders. In L. Verhoeven \& H. van Balkom (Eds.), Classification of developmental language disorders: Theoretical issues and clinical implications (pp. 235-260). Mahwah, NJ: Lawrence Erlbaum Associates.

Rosch, E., Mervis, C. B., Gray, W., Johnson, D., \& Boyes-Braem, P. (1976). Basic objects in natural categories. Cognitive Psychology, 8, 382-439.

Skinner, B. F. (1957). Verbal behavior. Acton, MA: Copley.

Tzuriel, D. (1992). The Children's Inferential Thinking Modifiability (CITM) test: Instruction manual. Ramat-Gan, Israel: Bar-Ilan University.

Tzuriel, D. (2003). Dynamic assessment. In J. W. Guthrie (Ed.), Encyclopedia of education (2nd ed., Vol. 1, pp. 127-131). New York NY: McMillan. 
Tzuriel, D. (2007). Transfer effects of teaching conceptual versus perceptual analogies. Journal of Cognitive Education and Psychology, 6(2), 194-217.

Tzuriel, D., \& Flor-Maduel, H. (2010). Prediction of early literacy by analogical thinking modifiability among kindergarten children. Journal of Cognitive Education and Psychology, 9, 207-227.

Tzuriel, D., \& George, T. (2009). Improvement of analogical reasoning and academic achievements by the Analogical Reasoning Program (ARP). Educational and Child Psychology, 29, 71-93.

Tzuriel, D., Haywood, H. C., \& Mandel, R. (2005). Effects of the sequence and pattern unit of Bright Start on seriational and math problem solving among kindergarten children of Ethiopian immigrants to Israel. Journal of Cognitive Education and Psychology, 5(1), 72-88.

Tzuriel, D., Kaniel, S., Kanner, A., \& Haywood, H. C. (1999). Effects of "Bright Start" program in kindergarten on transfer and academic achievement. Early Childhood Research Quarterly, 14, 111-141.

Tzuriel, D., Kaniel, S., Zeliger, M., Friedman, A., \& Haywood, H. C. (1998). Effects of the "Bright Start" program in kindergarten on use of mediated learning strategies and children's cognitive modifiability. Early Child Development and Care, 143, 1-20.

Tzuriel, D., \& Shamir, A. (2010). Mediation strategies and cognitive modifiability in young children as a function of peer mediation with young children (PMYC) program and training in analogies versus math tasks. Journal of Cognitive Psychology and Education, 9, 48-72.

Tzuriel, D., \& Stern, H. (2004). Understanding causality and understanding essentialism in super-ordinate categories among hearing impaired and hearing children (Doctoral dissertation). Ramat-Gan, Israel: Bar-Ilan University.

Vakil, E., Lifshitz, H., Tzuriel, D., Weiss, I., \& Arzuoan, Y. (2011). Analogies solving by individuals with and without intellectual disability: Different cognitive patterns as indicated by eye movements. Research in Developmental Disabilities, 32, 846-856.

Vygotsky, L. S. (1978). Mind in society: The development of higher psychological processes (Cole, M., John-Steiner, V., Scribner, S. \& Souberman, E., Eds.). Cambridge, MA: Harvard University Press. (Original work published 1934)

Younger, B. (1992). Developmental change in acquiring object kind concepts in infancy. Child Development, 63, 1526-1535.

Correspondence regarding this article should be directed to David Tzuriel, PhD, Bar-Ilan University, School of Education, Ramat-Gan, Israel, 5290002. E-mail: David.Tzuriel@biu.ac.il 